\title{
Serum cytoskeleton-associated protein 4 as a biomarker for the diagnosis of hepatocellular carcinoma
}

This article was published in the following Dove Medical Press journal: OncoTargets and Therapy

\author{
Yu Wangl,* \\ Weixin $\mathrm{Yu}^{1, *}$ \\ Mingqing $\mathrm{He}^{2, *}$ \\ Yan Huang ${ }^{3}$ \\ Mingyue Wang ${ }^{4}$ \\ Jinzhou Zhu ${ }^{5}$ \\ 'Department of General Surgery, \\ Jintan Affiliated Hospital of \\ Jiangsu University, Changzhou, \\ China; ${ }^{2}$ Department of Geriatrics, \\ The First Affiliated Hospital of \\ Soochow University, Suzhou, China; \\ ${ }^{3}$ Department of Infectious Diseases, \\ The First Affiliated Hospital of \\ Soochow University, Suzhou, China; \\ ${ }^{4}$ Department of Ultrasonography, \\ The First Affiliated Hospital of \\ Soochow University, Suzhou, China; \\ ${ }^{5}$ Department of Gastroenterology, The \\ First Affiliated Hospital of Soochow \\ University, Suzhou, China \\ *These authors contributed equally \\ to this work
}

Background: Alpha-fetoprotein (AFP) is the most commonly applied biomarker for diagnosis of hepatocellular carcinoma (HCC), but the low sensitivity and specificity limit its clinical application. Cytoskeleton-associated protein 4 (CKAP4) is a novel oncogenic protein involved in the development and progression of HCC. This study aimed to evaluate whether measurement of circulating CKAP4 could improve diagnostic accuracy for HCC.

Methods: We analyzed data for patients with HCC, chronic hepatitis B infection, and cirrhosis and healthy controls ( $\mathrm{n}=100$ in each group), recruited from two centers between July 2013 and December 2015. Circulating levels of CKAP4 were measured with commercial enzyme-linked immunosorbent assay kits. Receiver operating characteristics were used to evaluate diagnostic accuracy.

Results: Serum concentrations of CKAP4 were significantly elevated in the HCC group, in comparison with the three control groups (all $P<0.001$ ). The combined biomarker panel (AFP and CKAP4), created by binary logistic regression, presented better performance (area under the curve [AUC] $0.936,95 \%$ CI [0.908-0.965], sensitivity 0.800 , specificity 0.963 ) than AFP (AUC 0.875 [0.835-0.914], sensitivity 0.930 , specificity $0.430, P=0.001$ ) or CKAP4 (AUC 0.821 [0.776-0.866], sensitivity 0.790 , specificity $0.670, P<0.001$ ) alone to identify HCC, even though CKAP4 alone was not better than AFP $(P=0.093)$. Furthermore, the combined panel also presented a better performance even in identifying early HCC (AUC 0.922 [0.833-0.961]). Conclusion: Serum CKAP4 is a novel biomarker for HCC, and it could complement AFP in improving diagnostic accuracy.

Keywords: alpha-fetoprotein, biomarker, cytoskeleton-associated protein 4, hepatocellular carcinoma

\section{Introduction}

Hepatocellular carcinoma (HCC) is one of the most frequent causes of cancer-related death in the world. ${ }^{1,2}$ Approximately half of the global HCC morbidity and mortality occurs in China. ${ }^{1,3}$ Surgical resection and liver transplant only benefit patients with early-stage disease. ${ }^{4-6}$ Patients with more advanced disease are, unfortunately, only eligible for palliative therapy and usually present a poor prognosis, with median survival of 1-2 years. ${ }^{7}$ Given the situation, surveillance strategies for early tumor detection are urgently required in clinical practice. ${ }^{8}$

Imaging tools (eg, ultrasound and computed tomography) and serum biomarkers (eg, alpha-fetoprotein [AFP]) are commonly used to screen and diagnose HCC. ${ }^{9}$ However, it is still hard for imaging to differentiate small HCC from cirrhosis nodules. Meanwhile, evaluated levels of AFP are not only seen in patients with HCC but also in
Correspondence: Jinzhou Zhu; Mingyue Wang

The First Affiliated Hospital of Soochow University, 188 Shizi Street, Suzhou

215006, Jiangsu, China

$\mathrm{Tel}+86051265223637$

Fax +86051265223637

Email zhujinzhou@suda.edu.cn; wmy@suda.edu.cn
OncoTargets and Therapy 2019:12 359-364

359 
chronic hepatitis infections., ${ }^{3,10}$ Thus, there is an urgent need for the identification of novel biomarkers for HCC screening.

Cytoskeleton-associated protein 4 (CKAP4) is a $63-\mathrm{kDa}$ nonglycosylated and reversibly palmitoylated type II transmembrane protein. ${ }^{11,12}$ CKAP4 has recently been located in the endoplasmic reticulum, and identified as a cell surface receptor of the plasminogen activator and surfactant protein A, as well as an anti-proliferative factor to adversely mediate AKT signaling. ${ }^{13-15}$ Recently, Li et al ${ }^{16}$ examined the expression of CKAP4 in patients with HCC. They found that CKAP4 showed higher expression in HCC tumor tissues than in adjacent normal tissues and this was correlated with tumor size, intrahepatic metastases, portal-venous invasion, and HCC stage. Moreover, elevated CKAP4 expression was associated with favorable overall survival and longer disease-free survival. Recently, Li et al ${ }^{17}$ also proposed a novel mechanism for the mediation of EGFR/Gab1/AKT signaling activity, suggesting that CKAP4 is involved in progression and metastasis of HCC. Thus, these findings support CKAP4 playing a role in the oncogenic process of $\mathrm{HCC}$ and may be a potential target for diagnosis and therapy.

Recently, Yanagita et $\mathrm{al}^{11}$ designed an antibody that specifically recognized CKAP4 in serum. They found that the circulating levels of CKAP4 in patients with lung cancer were significantly higher than those in healthy controls. The hospital-based case-control study suggested serum CKAP4 as a potential biomarker for lung cancer.

In this study, our aim was to evaluate the serum levels of CKAP4 in patients with HCC and other liver diseases. Our team proposes that CKAP4 might be a potential diagnostic biomarker for HCC. The combination of serum biomarkers is recommended in cancer diagnosis, ${ }^{10,18}$ therefore we also tried to assess the diagnostic value of the combination of CKAP4 and AFP in the clinical management of HCC.

\section{Methods}

\section{Study population}

A total of 100 consecutive patients with HCC were recruited in this study from the First Affiliated Hospital of Soochow University, Suzhou and Jintan Affiliated Hospital of Jiangsu University, Changzhou, China (from July 2013 to December 2015). Meanwhile, we also recruited patients with chronic hepatitis $B(C H B)$ infection $(n=100)$ or cirrhosis $(n=100)$ from the two hospitals and healthy controls $(n=100)$ from the Out-patient Department. Written informed consent was obtained from each participant. This study was approved by the Ethics Committee of each center, in accordance with the 1964 Helsinki Declaration and its later amendments or comparable ethical standards.

\section{Inclusion criteria}

HCC was firstly diagnosed by imaging (eg, ultrasound or computed tomography), and then the cases were further confirmed by histopathology, based on the guidelines of the American Association for the Study of Liver Diseases. ${ }^{19} \mathrm{HCC}$ stages were classified according to the Barcelona Clinic Liver Cancer staging system. ${ }^{7}$ In this study, tumors at Barcelona Clinic Liver Cancer stage 0 and A were categorized as earlystage HCC.

The diagnosis of CHB infection was based on: 1) hepatitis B surface antigen-positive for the previous 6 months; 2) hepatitis B virus DNA $>10^{3}$ copies/mL; and 3) elevated alanine aminotransferase, according to the guidelines for prevention and treatment of chronic HBV infection. ${ }^{20}$

Cirrhosis was diagnosed based on the histopathology of liver biopsy samples, and on other evidence (eg, portal hypertension, splenomegaly, nodular liver contour, presence of ascites, varices, enlargement of the caudate lobe, and collateral portal-venous anastomoses). ${ }^{19}$

The inclusion criteria for healthy controls were the following: 1) normal liver biochemistry; 2) no history of liver disease; 3) no other systematic diseases; and 4) no malignant disease.

\section{Laboratory examination}

Serum samples of patients were collected at the time of diagnosis. All samples were centrifuged and stored at $-80^{\circ} \mathrm{C}$ for further evaluation. Serum biochemical tests were performed using a Hitachi 7600 Auto-Analyzer (Hitachi Ltd., Tokyo, Japan). Circulating levels of CKAP4 were measured with commercial enzyme-linked immunosorbent assay kits (Catalog No CSB-EL005453HU; CUSABIO, Wuhan, China). The intra-assay and inter-assay coefficients of variation of serum CKAP4 measurement were $7.2 \%$ and $9.7 \%$, respectively. Circulating levels of AFP were measured by chemiluminescence immunoassay with an Abbott-Architect Immunoanalyzer (Abbott Laboratories, Abbott Park, IL, USA). The examination was performed blinded and in duplicate.

\section{Statistical analyses}

Differences among various groups (more than two) used oneway analysis of variance or the Kruskal-Wallis test. Receiver operating characteristic curves were developed to evaluate 
sensitivity, specificity, and areas under the curves (AUCs) with $95 \%$ CI. The optimum cutoff values for diagnosis were calculated according to the Youden Index. To investigate whether the combined utility of biomarkers was better than either alone, a new variable of predicted probability $(p)$ for $\mathrm{HCC}$ was created on the basis of an equation obtained by binary logistic regression (all HCC vs Control + Cirrhosis + CHB). All statistical analyses and plotting were performed using Stata (version MP 11.2; StataCorp LP, College Station, TX, USA), Prism (version 6; GraphPad Software, Inc., LA Jolla, CA, USA), and SPSS (version 24.0; IBM Corporation, Armonk, NY, USA). A two-sided $P<0.05$ was considered statistically significant.

\section{Results}

The characteristics of all participants and HCC patients are indicated in Tables 1 and 2.

\section{Biomarker levels}

As shown in Figure 1A, serum AFP in patients with HCC (median 148.2 [IQR 48.2-277.5] ng/mL) presented higher levels than those in controls (healthy control: 7.4 [4.8-267.3] $\mathrm{ng} / \mathrm{mL}$; CHB: 31.5 [22.7-52.2] ng/mL; cirrhosis: 31.3 [22.2-53.3] ng/mL; $P<0.0001)$.

Serum levels of CKAP4 were significantly higher in patients with $\mathrm{HCC}(345.9$ [264.8-474.5] pg/mL) than in all controls (healthy control: 184.2 [111.1-267.3] pg/mL; CHB: 180.1 [97.2-283.5] pg/mL; cirrhosis: 228.1 [129.2-307.1] $\mathrm{pg} / \mathrm{mL}$; all $P<0.001)$, as shown in Figure 1B.

\section{Cutoff values and AUCs}

According to the guideline, ${ }^{21}$ we chose $20 \mathrm{ng} / \mathrm{mL}$ as the cutoff value for AFP in this study (AUC 0.875 [95\% CI $0.835-0.914]$, sensitivity 0.930 , specificity 0.430 ; Figure $2 \mathrm{~A}$ and Table 3 ). Receiver operating characteristic curves showed that the optimum diagnostic cutoff value for CKAP4 was $250.15 \mathrm{pg} / \mathrm{mL}$ (AUC 0.821 [0.776-0.866], sensitivity 0.790, specificity 0.670). The comparison between APF and CKAP4 presented no difference $(P=0.093)$.

\section{Combined biomarkers}

To assess the diagnostic value of the combined variables, a new variable of predicted probability $(p)$ for HCC was developed on the equation obtained from the binary logistic regression (all HCC vs all controls). The equation was as follows:

$$
\ln \left(\frac{p}{1-p}\right)=0.021 \times \mathrm{AFP} 0.015 \times \mathrm{CKAP} 4-6.927
$$

In Figure 2A, the optimum cutoff value calculated from the combined variables was 0.404 . This indicated a better predicting ability (AUC 0.936 [0.908-0.965], sensitivity 0.800, specificity 0.963), compared with AFP and CKAP4 $(P=0.001$ and $P<0.001)$. Predictive values and likelihood ratios for AFP, CKAP4, and the combination in the diagnosis of HCC are shown in Table 3.

\section{Early-stage HCC}

Given that early detection is the key to improve prognosis of patients with HCC, the diagnostic ability of AFP, CKAP4,

Table I Characteristics of participants

\begin{tabular}{l|l|l|l|l}
\hline Items & Control & CHB & Cirrhosis & HCC \\
\hline Age (years) & $57.2 \pm 6.8$ & $54.1 \pm 6.4$ & $57.0 \pm 7.2$ & $55.0 \pm 6.2$ \\
Sex & 84 & 84 & 88 & 84 \\
$\quad$ Male & 16 & 16 & 12 & 16 \\
$\quad$ Female & $22.7 \pm 3.2$ & $23.4 \pm 3.9$ & $22.1 \pm 2.9$ & $22.3 \pm 3.3$ \\
BMI & $22.0(16.0-34.0)$ & $48.0(32.0-62.0)$ & $51.0(33.0-67.0)$ & $67.0(43.0-85.5)$ \\
ALT (U/L) & $39.0(12.0-48.0)$ & $51.0(29.0-56.0)$ & $78.0(40.0-98.0)$ & $99.0(64.0-127.0)$ \\
GGT (U/L) & & & & 85 \\
HBsAg & 100 & 100 & 84 & 15 \\
$\quad$ Positive & & 0 & 16 & 81 \\
$\quad$ Negative & NA & NA & 77 & 19 \\
Child-Pugh & NA & NA & 23 & $11(9-14)^{*}$ \\
A & B & & $11(9-15)$ & \\
MELD score & & & \\
\hline
\end{tabular}

Notes: Data presented as mean $\pm \mathrm{SD}$, median (IQR), or number. Each group, $\mathrm{n}=100$. *MELD score only in HCC cirrhosis $(+)$ patients $(\mathrm{n}=9 \mathrm{I})$.

Abbreviations: ALT, alanine aminotransferase; BMI, body mass index; CHB, chronic hepatitis B; GGT, gamma-glutamyltranspeptidase; HBsAg, hepatitis B surface antigen; HCC, hepatocellular carcinoma; MELD, Model for End-Stage Liver Disease; NA, not applicable. 
Table 2 Tumor features of HCC patients

\begin{tabular}{l|l}
\hline Items & HCC $(\mathbf{n}=\mathbf{1 0 0})$ \\
\hline Tumor size $(\mathrm{cm})$ & \\
$<3$ & 28 \\
$3-5$ & 49 \\
$>5$ & 23 \\
Tumor number & 71 \\
Single & 29 \\
Multiple & \\
BCLC stage & 7 \\
0 & 56 \\
A & 18 \\
B & 19 \\
C & 0 \\
D & \\
\hline
\end{tabular}

Abbreviations: BCLC, Barcelona Clinic Liver Cancer; HCC, hepatocellular carcinoma.

and the combination in early-stage HCC were further evaluated. As shown in Figure $2 \mathrm{~B}$ and Table 3, the combination presented the best AUC (0.922 [0.833-0.961]), followed by AFP (0.834 [0.782-0.886], $P<0.001)$ and CKAP4 (0.842 [0.792-0.893], $P=0.001)$. The latter two still indicated no difference $(P=0.831)$.

\section{Discussion}

Recent evidence reported that the hepatic expression of CKAP4 increased in patients with HCC, and that CKAP4 is associated with clinical outcome and prognosis. ${ }^{16,17}$ Kimura et $\mathrm{al}^{12}$ reported that CKAP4 at the plasma membrane stimulates proliferation of several cell lines, including pancreatic cancer cells and lung cancer cells, via activating the phosphoinositide 3-kinase-AKT pathway. Furthermore, they found that the expression of CKAP4 in pancreatic and lung cancers was related to a poor prognosis for patients. Shinno et $\mathrm{al}^{22}$ reported that overexpression of CKAP4 could be observed in about $40 \%$ of esophageal squamous cell carcinoma patients. The phosphoinositide 3-kinase-AKT pathway may be a downstream cascade of the Dickkopf WNT signaling pathway inhibitor 1-CKAP4 signaling axis in esophageal squamous cell carcinoma cases. The role and mechanisms of CKAP4 in cancers still need further investigation.

Circulating biomarkers are promising potential tools for screening and early detection of HCC, owing to their noninvasive and cost-efficient features. ${ }^{23,24}$ AFP is currently still the most widely used biomarker in HCC, even though it presents a series of disadvantages: 1) less specificity, especially in patients with chronic hepatitis or cirrhosis; and 2) associated with tumor size and not suitable for surveillance at an early stage. ${ }^{23}$ Therefore, a new reliable biomarker to complement AFP is urgently required to improve early detection.

In this study, we investigate whether measurement of circulating CKAP4 levels could improve diagnostic accuracy for $\mathrm{HCC}$, in a hospital-based case-control study that included $100 \mathrm{HCC}$ patients and 300 non-HCC controls (ie, 100 healthy controls, 100 controls with chronic HBV infection, and 100 controls with cirrhosis). Our study followed the widely accepted protocol for evaluation of diagnostic biomarkers. ${ }^{25}$ It found that serum concentrations of
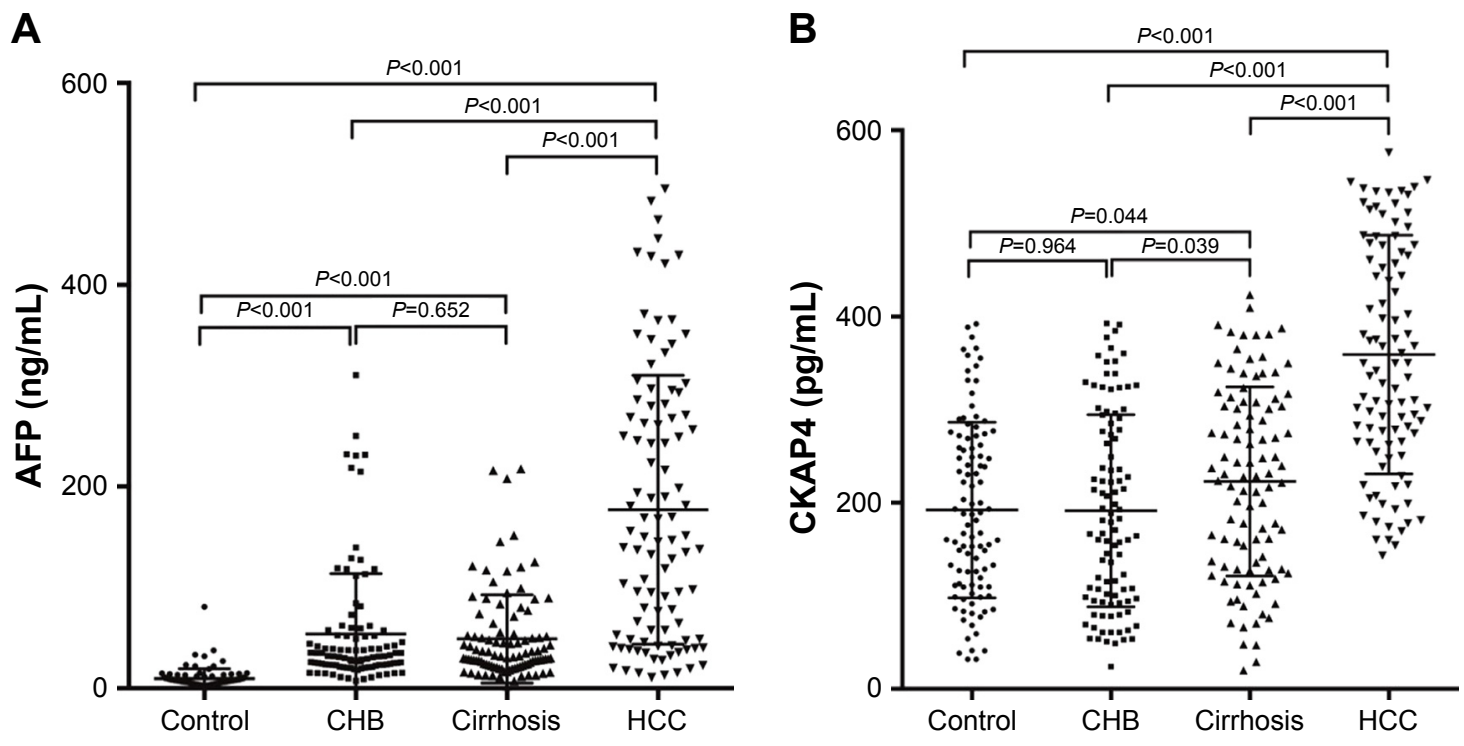

Figure I Circulating concentrations of AFP and CKAP4 in HCC, control, CHB, and cirrhosis groups.

Notes: (A) Levels of serum AFP ( $\mathrm{ng} / \mathrm{mL}$, median [IQR]); (B) levels of serum CKAP4 (pg/mL, median [IQR]).

Abbreviations: AFP, alpha-fetoprotein; CHB, chronic hepatitis B; CKAP4, cytoskeleton-associated protein 4; HCC, hepatocellular carcinoma. 

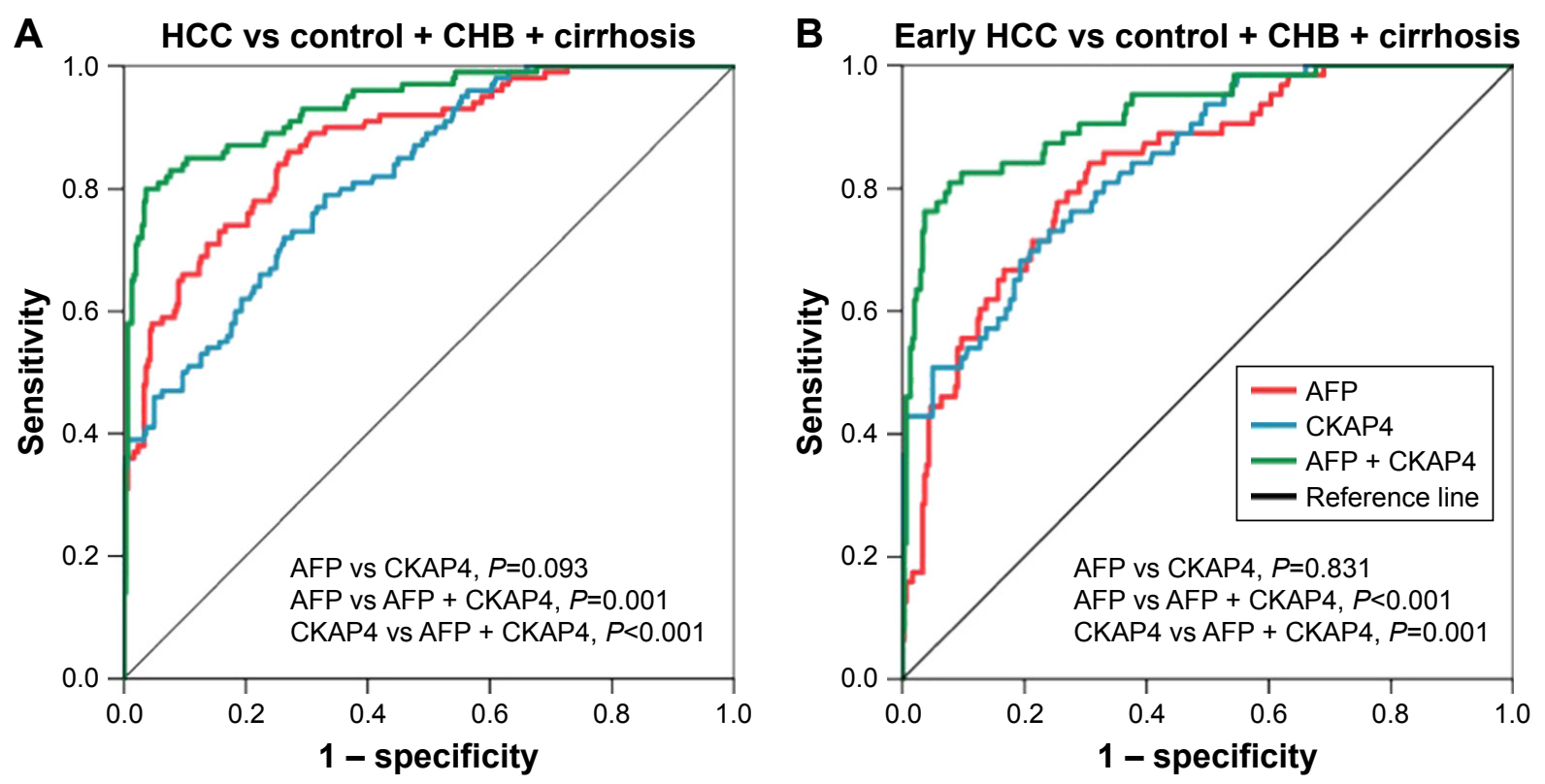

Figure 2 ROC curves for AFP, CKAP4, and the combination in the diagnosis of HCC and early-stage HCC.

Note: (A) ROC curves for AFP, CKAP4, and the combinations for patients with HCC vs all controls; (B) ROC curves for AFP, CKAP4, and the combinations for patients with early-stage $\mathrm{HCC}$ vs all controls.

Abbreviations: AFP, alpha-fetoprotein; CHB, chronic hepatitis B; CKAP4, cytoskeleton-associated protein 4; HCC, hepatocellular carcinoma; ROC, receiver operating characteristic.

CKAP4 were significantly elevated in the HCC group, in comparison with the three control groups. Besides, $86 \%$ of AFP-negative patients with $\mathrm{HCC}$ also indicated increased serum levels of CKAP4. The combined biomarker panel (AFP and CKAP4), created by binary logistic regression, outperformed stand-alone assays of AFP or CKAP4 to identify HCC, but CKAP4 alone was not better than AFP. The combined panel presented good diagnostic accuracy, even in identifying early HCC. However, the molecular mechanism and clinical importance of CKAP4 in HCC still need to be further explored.

Our study has some limitations. To begin with, patients with other digestive cancers (eg, cholangiocarcinoma and gastric cancer) should be included to rule out potential falsepositive results. Given the previous findings, the serum levels of CKAP4 in lung cancer, ${ }^{11}$ renal cancer, ${ }^{26}$ and esophageal cancer ${ }^{22}$ should be evaluated in further studies to assess the specificity. Also, validation studies should be conducted in patients with HCV-induced or alcohol-induced HCC, which are prevalent in western countries. Furthermore, this study was of a retrospective design and thus large prospective studies to assess the role of CKAP4 in the development of HCC are needed.

\section{Conclusion}

This is the first study to report the levels of serum CKAP4 in liver diseases. Our result indicates that serum CKAP4 could potentially be used to identify HCC. The result also shows that the combination of CKAP4 and AFP could enhance accuracy and specificity in the diagnosis of $\mathrm{HCC}$,

Table 3 Performance of AFP, CKAP4, and the combination in the diagnosis of HCC and early-stage HCC

\begin{tabular}{l|l|l|l|l|l|l|l|l}
\hline Items & AUC & $\mathbf{9 5 \%} \mathbf{C l}$ & Sensitivity & Specificity & PPV & NPV & LR+ & LR- \\
\hline HCC vs control/CHB/cirrhosis & & & & & & & & \\
$\quad$ AFP & 0.875 & $0.835-0.914$ & 0.930 & 0.430 & 0.352 & 0.949 & 1.632 & 0.163 \\
CKAP4 & 0.821 & $0.776-0.866$ & 0.790 & 0.670 & 0.444 & 0.905 & 2.394 & 0.313 \\
$\quad$ Combination & 0.936 & $0.908-0.965$ & 0.800 & 0.963 & 0.879 & 0.935 & 21.818 & 0.208 \\
Early HCC vs control/CHB/cirrhosis & & & & & & \\
AFP & 0.834 & $0.782-0.886$ & 0.905 & 0.430 & 0.250 & 0.956 & 1.587 & 0.221 \\
CKAP4 & 0.842 & $0.792-0.893$ & 0.810 & 0.670 & 0.340 & 0.944 & 2.453 & 0.284 \\
Combination & 0.922 & $0.883-0.961$ & 0.762 & 0.963 & 0.814 & 0.951 & 20.779 & 0.247 \\
\hline
\end{tabular}

Abbreviations: AFP, alpha-fetoprotein; AUC, area under receiver operating characteristic curve; CHB, chronic hepatitis B; CKAP4, cytoskeleton-associated protein 4; HCC, hepatocellular carcinoma; LR, likelihood ratio; NPV, negative predictive value; PPV, positive predictive value. 
even early-stage HCC, and may provide a new diagnostic strategy for HCC patients.

\section{Acknowledgments}

We thank the participants and relevant research staff for their contributions to the study. This study was funded by the Science and Technology Program of Medicine and Health of Jintan City.

\section{Disclosure}

The authors report no conflicts of interest in this work.

\section{References}

1. Torre LA, Bray F, Siegel RL, Ferlay J, Lortet-Tieulent J, Jemal A. Global cancer statistics, 2012. CA Cancer J Clin. 2015;65(2):87-108.

2. Bertuccio P, Turati F, Carioli G, et al. Global trends and predictions in hepatocellular carcinoma mortality. J Hepatol. 2017;67(2):302-309.

3. Luo P, Yin P, Hua R. A Large-scale, multicenter serum metabolite biomarker identification study for the early detection of hepatocellular carcinoma. Hepatology. 2018;67(2):662-675.

4. Gerbes A, Zoulim F, Tilg H, et al. Gut roundtable meeting paper: selected recent advances in hepatocellular carcinoma. Gut. 2018;67(2):380-388.

5. Ligat G, Schuster C, Baumert TF. HBV core variants, liver fibrosis and hepatocellular carcinoma. Hepatology. Epub 2018 Aug 23.

6. Tzartzeva K, Obi J, Rich NE, et al. Surveillance imaging and alpha fetoprotein for early detection of hepatocellular carcinoma in patients with cirrhosis: a meta-analysis. Gastroenterology. 2018;154(6):1706-1718e1.

7. Llovet JM, Di Bisceglie AM, Bruix J, et al; Panel of Experts in HCCDesign Clinical Trials. Design and endpoints of clinical trials in hepatocellular carcinoma. J Natl Cancer Inst. 2008;100(10):698-711.

8. Shoreibah MG, Bloomer JR, McGuire BM, Massoud OI. Surveillance for hepatocellular carcinoma: evidence, guidelines and utilization. Am J Med Sci. 2014;347(5):415-419.

9. Clavien PA, Lesurtel M, Bossuyt PM, et al; OLT for HCC Consensus Group. Recommendations for liver transplantation for hepatocellular carcinoma: an international consensus conference report. Lancet Oncol. 2012;13(1):e11-e22.

10. Marrero JA, Feng Z, Wang Y, et al. Alpha-fetoprotein, des-gamma carboxyprothrombin, and lectin-bound alpha-fetoprotein in early hepatocellular carcinoma. Gastroenterology. 2009;137(1):110-118.

11. Yanagita K, Nagashio R, Jiang SX, et al. Cytoskeleton-associated protein 4 is a novel serodiagnostic marker for lung cancer. Am J Pathol. 2018; 188(6):1328-1333.
12. Kimura H, Fumoto K, Shojima K, et al. CKAP4 is a Dickkopf1 receptor and is involved in tumor progression. J Clin Invest. 2016;126(7): 2689-2705.

13. Kazi AS, Tao JQ, Feinstein SI, Zhang L, Fisher AB, Bates SR. Role of the PI3-kinase signaling pathway in trafficking of the surfactant protein A receptor P63 (CKAP4) on type II pneumocytes. Am J Physiol Lung Cell Mol Physiol. 2010;299(6):L794-L807.

14. Conrads TP, Tocci GM, Hood BL, et al. CKAP4/p63 is a receptor for the frizzled-8 protein-related antiproliferative factor from interstitial cystitis patients. $J$ Biol Chem. 2006;281(49):37836-37843.

15. Matika CA, Wasilewski M, Arnott JA, Planey SL. Antiproliferative factor regulates connective tissue growth factor $(\mathrm{CTGF} / \mathrm{CCN} 2)$ expression in T24 bladder carcinoma cells. Mol Biol Cell. 2012;23(10): 1976-1985.

16. Li SX, Tang GS, Zhou DX, et al. Prognostic significance of cytoskeleton-associated membrane protein 4 and its palmitoyl acyltransferase DHHC2 in hepatocellular carcinoma. Cancer. 2014;120(10): $1520-1531$

17. Li SX, Liu LJ, Dong LW, et al. CKAP4 inhibited growth and metastasis of hepatocellular carcinoma through regulating EGFR signaling. Tumour Biol. 2014;35(8):7999-8005.

18. Marrero JA, Lok AS. Newer markers for hepatocellular carcinoma. Gastroenterology. 2004;127(5 Suppl 1):S113-S119.

19. Bruix J, Sherman M; Practice Guidelines Committee, American Association for the Study of Liver Diseases. Management of hepatocellular carcinoma. Hepatology. 2005;42(5):1208-1236.

20. Lok AS, McMahon BJ. Chronic hepatitis B: update 2009. Hepatology. 2009;50(3):661-662.

21. El-Serag HB, Marrero JA, Rudolph L, Reddy KR. Diagnosis and treatment of hepatocellular carcinoma. Gastroenterology. 2008;134(6): $1752-1763$.

22. Shinno N, Kimura H, Sada R, et al. Activation of the Dickkopf1-CKAP4 pathway is associated with poor prognosis of esophageal cancer and anti-CKAP4 antibody may be a new therapeutic drug. Oncogene. 2018;37(26):3471-3484.

23. Forner A, Bruix J. Biomarkers for early diagnosis of hepatocellular carcinoma. Lancet Oncol. 2012;13(8):750-751.

24. Zhang K, Song P, Gao J, Li G, Zhao X, Zhang S. Perspectives on a combined test of multi serum biomarkers in China: towards screening for and diagnosing hepatocellular carcinoma at an earlier stage. Drug Discov Ther. 2014;8(3):102-109.

25. Bossuyt PM, Reitsma JB, Bruns DE, et al; Standards for Reporting of Diagnostic Accuracy. Towards complete and accurate reporting of studies of diagnostic accuracy: the STARD initiative. Ann Intern Med. 2003;138(1):40-44.

26. Sun CM, Geng J, Yan Y, Yao X, Liu M. Overexpression of CKAP4 is associated with poor prognosis in clear cell renal cell carcinoma and functions via cyclin B signaling. J Cancer. 2017;8(19):4018-4026.
OncoTargets and Therapy

\section{Publish your work in this journal}

OncoTargets and Therapy is an international, peer-reviewed, open access journal focusing on the pathological basis of all cancers, potential targets for therapy and treatment protocols employed to improve the management of cancer patients. The journal also focuses on the impact of management programs and new therapeutic agents and protocols on

\section{Dovepress}

patient perspectives such as quality of life, adherence and satisfaction. The manuscript management system is completely online and includes a very quick and fair peer-review system, which is all easy to use. Visit http://www.dovepress.com/testimonials.php to read real quotes from published authors. 